\title{
SINR Analysis of Cognitive Underlay Systems with Multiple Primary Transceivers in Nakagami-m Fading
}

\author{
Petros S. Bithas*, Athanasios A. Rontogiannis* and Kostas Berberidis ${ }^{\dagger}$ \\ * IAASARS, National Observatory of Athens, GR-15236, Penteli, Greece \\ $\dagger$ Dept. of Computer Engineering and Informatics, University of Patras, 26500 Rio-Patras, Greece \\ Email:\{pbithas@ieee.org, tronto@noa.gr, berberid@ceid.upatras.gr\}
}

\begin{abstract}
In this paper the outage probability of a cognitive decode-and-forward relay network operating over Nakagami- $m$ fading channels is evaluated. Based on the underlay approach, secondary transmissions are allowed only in cases where interference constraints on the primary destination receivers are satisfied. Additionally, by taking into consideration the interfering effects coming from the multiple primary transmitters, the signal-tointerference-plus-noise-ratio statistic of the secondary nodes is investigated. The derived results include exact expressions as well as approximated ones for high values of the maximum allowed transmitted power at the cognitive network and/or the interference limited case. We present numerical performance evaluation results for various channel conditions and communication scenarios. These results are complemented by equivalent computer simulated ones, which validate the accuracy of the proposed analysis.
\end{abstract}

Index Terms-Cognitive relay networks, interference constraints, multiple primary users, Nakagami- $m$ fading, signal-tointerference-plus-noise-ratio.

\section{INTRODUCTION}

In the last years, a tremendous growth on the wireless communication demands has been observed. The main reasons for this development are the requirements for higher data rates and the exponential increasing number of connected devices. A promising approach that is expected to improve this situation, by increasing spectrum efficiency and thus the overall network throughput, is cognitive radio. In cognitive network, secondary users are allowed to simultaneously operate, under the same frequency bands, with the primary ones, but constrained in all cases by a minimum disruption on the later. In this context, in order to protect licensed (primary) users data transmission, various approaches have been proposed, such as spectrum underlay, overlay and interweave [1]. As far as the underlay approach is concerned, the basic idea is that as long as the interference generated by the secondary users does not exceed a predefined threshold at the primary destinations, secondary's network transmissions are allowed [2]. Additionally, in order to extend the coverage of secondary networks, and thus further enhance their reliability, cooperative relaying techniques have been employed, giving rise to a new research field that is known as cognitive relay networks (CRN), which is also the subject of the current work.

The research area of underlay $\mathrm{CRN}$ has recently gained a considerable attention, which is proved by the numerous contributions that have appeared lately, e.g., [3]-[7]. More specifically, in [3], the outage probability (OP) of an underlay based CRN, which supports a selection criterion, is investigated. Additionally, in [4], the OP of a dual-hop decode-andforward (DF) CRN operating over Rayleigh fading channels is studied in the presence of interference by a single primary source. However, the existence of a single primary user is idealized and thus the research soon focused to more realistic scenarios, where multiple primary users exist, e.g., [8], [9]. In [8], the analysis was extended to include the impact of multiple primary transmissions on the OP of a cognitive DF (single) relay network. A general remark on these works is that despite the fact that they have provided clear contribution on this topic, Rayleigh fading channels have been assumed. Therefore, only a specific channel fading situation has been studied, i.e., the worst scenario, and thus the presented OP results represent a limiting case. This research gap has been recently fulfilled by several researchers e.g., [10]-[12]. However, none of these works takes into consideration the impact of the multiple primary users' interference on the secondary network, which motivates our work.

In this work, we assume Nakagami- $m$ fading for the wireless links of the primary network, consisting of multiple transceivers, and the cooperative single relay DF secondary network. In this context, new exact and asymptotic analytical expressions for the cumulative distribution function (CDF) of the output signal-to-interference-plus-noise-ratio (SINR) at the destination node of the cognitive network, are derived. In addition, considering higher values of the maximum available transmit power as well as an interference limiting case, simplified asymptotic expressions are also extracted. The rest of the paper is organized as follows. Section II contains the general description of the system and channel model under consideration. In Section III, a generic OP analysis is presented accompanied by two special cases, where simplified results 


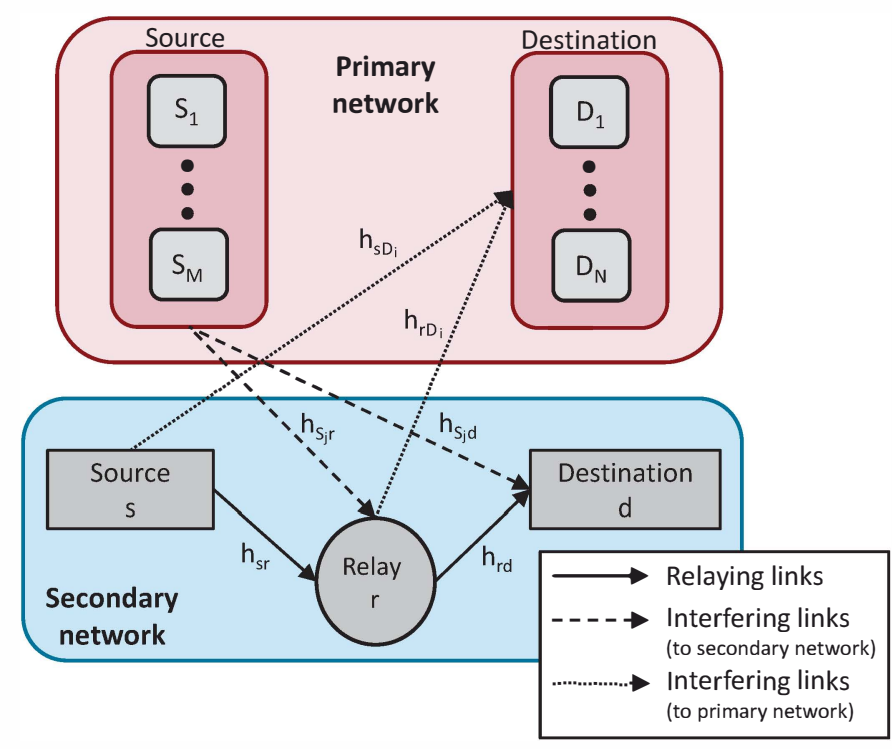

Fig. 1. The mode of operation of the underlay cognitive network under consideration.

are presented. Section IV presents some numerical results and Section V includes the concluding remarks of this paper. For the reader's convenience, most of the notations and symbols used in the rest of this paper are summarized in Table I.

\section{System AND Channel Model}

We consider a dual-hop CRN, where primary users coexist with secondary ones, as shown in Fig. 1. At the first phase, the secondary source $\mathrm{s}$ transmits a signal only to the relay $\mathrm{r}$, while the direct path between $\mathrm{s}$ and the secondary destination $\mathrm{d}$ is considered to be blocked. At the second phase, the relay node forwards the re-encoded version of the transmitted signal to d. Additionally, all secondary transmissions are allowed as long as the generated interference on the set of primary destinations $\mathrm{D}_{i}$ 's, with $i=1, \ldots, N$, remains below an interference threshold $I$ [3]. Thus, the transmit powers at $\mathrm{s}$ and $r$ should be constrained as follows [13]

$$
\begin{gathered}
P_{\mathrm{s}}=\min \left\{\frac{I}{\max _{i=1, \ldots, N}\left(\left|h_{\mathrm{sD}_{i} \mid}\right|^{2}\right)}, P_{\max }\right\} \\
P_{\mathrm{r}}=\min \left\{\frac{I}{\max _{i=1, \ldots, N}\left(\left|h_{\mathrm{rD}_{i}}\right|^{2}\right)}, P_{\max }\right\} .
\end{gathered}
$$

Additionally, due to the simultaneous transmissions from the primary sources $\mathrm{S}_{j}$ 's, the received signals at $\mathrm{r}$ and $\mathrm{d}$ are also subject to interfering effects. The corresponding received instantaneous SINRs are given by

$$
\begin{gathered}
\hat{\gamma}_{\mathrm{sr}}=\frac{P_{\mathrm{s}}\left|h_{\mathrm{sr}}\right|^{2}}{\sum_{j=1}^{M} P_{T}\left|h_{\mathrm{S}_{j} \mathrm{r}}\right|^{2}+N_{0}} \\
\hat{\gamma}_{\mathrm{rd}}=\frac{P_{\mathrm{r}}\left|h_{\mathrm{rd}}\right|^{2}}{\sum_{j=1}^{M} P_{T}\left|h_{\mathrm{S}_{j} \mathrm{~d}}\right|^{2}+N_{0}} .
\end{gathered}
$$

TABLE I

NOTATION AND SYMBOLS

\begin{tabular}{|r|l|}
\hline$h_{\mathrm{SD}_{i}}$ & Channel gain between $\mathrm{s}$ and $\mathrm{D}_{i}$ \\
\hline$h_{\mathrm{rD}_{i}}$ & Channel gain between $\mathrm{r}$ and $\mathrm{D}_{i}$ \\
\hline$h_{\mathrm{S} j \mathrm{r}}$ & Channel gain between $\mathrm{S}_{j}$ and $\mathrm{r}$ \\
\hline$h_{\mathrm{S}_{j} \mathrm{~d}}$ & Channel gain between $\mathrm{S}_{j}$ and $\mathrm{d}$ \\
\hline$h_{\mathrm{sr}}$ & Channel gain between $\mathrm{s}$ and $\mathrm{r}$ \\
\hline$h_{\mathrm{rd}}$ & Channel gain between $\mathrm{r}$ and $\mathrm{d}$ \\
\hline$P_{\max }$ & Maximum allowable transmit power at $\mathrm{s}$ and $\mathrm{r}$ \\
\hline$I$ & $\begin{array}{l}\text { Maximum tolerable interference level at which } \\
\text { D }_{i} \text { 's can still maintain reliable communication }\end{array}$ \\
\hline$P_{T}$ & Transmit power at $\mathrm{S}_{j}$ 's \\
\hline$N_{0}$ & Noise power \\
\hline$M$ & Number of primary source nodes \\
\hline$N$ & Number of primary destination nodes \\
\hline
\end{tabular}

It is assumed here that $\left|h_{\mathrm{SD}_{i}}\right|^{2},\left|h_{\mathrm{rD}_{i}}\right|^{2},\left|h_{\mathrm{S}_{j} \mathrm{r}}\right|^{2},\left|h_{\mathrm{S}_{j} \mathrm{~d}}\right|^{2},\left|h_{\mathrm{sr}}\right|^{2}$, $\left|h_{\text {rd }}\right|^{2}$ follow the gamma distribution with probability density function (PDF) given by

$$
f_{\left|h_{X}\right|^{2}}(y)=\frac{m_{X}^{m_{X}} y^{m_{X}-1}}{\bar{\gamma}_{X}^{m_{X}} \Gamma\left(m_{X}\right)} \exp \left(-\frac{m_{X}}{\bar{\gamma}_{X}} y\right)
$$

where $m_{X}, \bar{\gamma}_{X}$ denote the shaping parameter and the mean value of the distribution, with $X \in\left\{\mathrm{sD}_{i}, \mathrm{rD}_{i}, \mathrm{~S}_{j} \mathrm{r}, \mathrm{S}_{j} \mathrm{~d}, \mathrm{sr}, \mathrm{rd}\right\}$ and $\Gamma(\cdot)$ is the Gamma function [14, eq. (8.310/1)]. Assuming integer values for $m_{X}$, the corresponding CDF can be expressed as

$$
F_{\left|h_{X}\right|^{2}}(y)=1-\exp \left(-\frac{m_{X}}{\bar{\gamma}_{X}} y\right) \sum_{k=0}^{m_{X}-1}\left(\frac{m_{X}}{\bar{\gamma}_{X}}\right)^{k} \frac{y^{k}}{k !}
$$

For the DF protocol under consideration, due to the imperfect detection at the relay, correctly (or not) decoded signals are forwarded to $d$. Thus, the $s-r-d$ instantaneous output SINR is given by $\gamma_{\text {out }}=\min \left\{\hat{\gamma}_{\mathrm{sr}}, \hat{\gamma}_{\mathrm{rd}}\right\}$ and the corresponding CDF by [15]

$$
F_{\gamma_{\text {out }}}(\gamma)=1-\left[1-F_{\hat{\gamma}_{\text {sr }}}(\gamma)\right]\left[1-F_{\hat{\gamma}_{\text {rd }}}(\gamma)\right] .
$$

In the next section, analytical expressions for the CDF of $\hat{\gamma}_{\mathrm{sr}}$ and $\hat{\gamma}_{\text {rd }}$ will be provided. Substituting these expressions in (7), the OP, which is defined as the probability that the SINR falls below a predefined threshold $\gamma_{\mathrm{th}}$, can be directly evaluated as $P_{\text {out }}=F_{\gamma_{\text {out }}}\left(\gamma_{\text {th }}\right)$.

\section{Outage Probability Analysis}

In this section, we investigate the CDF of $\hat{\gamma}_{\mathrm{sr}}, \hat{\gamma}_{\mathrm{rd}}$. First, we derive an exact generic expression for these CDFs, while, next, simplified expressions are provided for two special cases. We start by defining the following new random variable

$$
U=\min \left\{\frac{I}{\max _{i=1, \ldots, N}\left(\left|h_{\mathrm{sD}_{i}}\right|^{2}\right)}, P_{\max }\right\}\left|h_{\mathrm{sr}}\right|^{2} .
$$


The CDF of $U$ can be expressed as

$$
\begin{aligned}
F_{U}(x)= & \underbrace{\operatorname{Pr}\left(\frac{I}{Y}\left|h_{\mathrm{sr}}\right|^{2} \leq x, Y>\frac{I}{P_{\max }}\right)}_{\mathcal{F}_{1}(x)} \\
& +\underbrace{\operatorname{Pr}\left(P_{\max }\left|h_{\mathrm{sr}}\right|^{2} \leq x, Y \leq \frac{I}{P_{\max }}\right)}_{\mathcal{F}_{2}(x)}
\end{aligned}
$$

where $Y=\max _{i=1, \ldots, N}\left(\left|h_{\mathrm{SD}_{i}}\right|^{2}\right)$. In the following analysis, we have assumed independent and identical distributed (i.i.d.) fading conditions, i.e., $m_{\mathrm{sD}_{i}}=m_{\mathrm{sD}}, \bar{\gamma}_{\mathrm{sD}_{i}}=\bar{\gamma}_{\mathrm{sD}}, m_{\mathrm{S}_{j} \mathrm{r}}=m_{\mathrm{Sr}}$, $\bar{\gamma}_{\mathrm{S}_{j} \mathrm{r}}=\bar{\gamma}_{\mathrm{Sr}}, \forall i, j$, and integer values of $m_{\mathrm{SD}}$. Thus, using first the binomial theorem [14, eq. (1.111)] and then the multinomial identity [16, eq. (24.1.2)], the CDF of $Y$ can be expressed as

$$
F_{Y}(y)=\mathcal{S}_{1}(0) y^{\theta} \exp \left(-\frac{m_{\mathrm{sD}} i}{\bar{\gamma}_{\mathrm{sD}}} y\right)
$$

where

$$
\mathcal{S}_{1}(\alpha)=\sum_{i=\alpha}^{N}\left(\begin{array}{c}
N \\
i
\end{array}\right)(-1)^{i} \sum_{\substack{i_{0}, i_{1}, \ldots, i_{m_{\mathrm{sD}}-1}=0 \\
i_{0}+\ldots+i_{m_{\mathrm{sD}}-1}=i}}^{i} \frac{i !\left(m_{\mathrm{sD}} / \bar{\gamma}_{\mathrm{sD}}\right)^{\theta}}{\left[\prod_{k=0}^{m_{\mathrm{sD}}-1} i_{k} !(k !)^{i_{k}}\right]}
$$

and $\theta=\sum_{k=0}^{m_{\mathrm{sD}}-1} k i_{k}$. The corresponding PDF expression is given by

$$
\begin{aligned}
f_{Y}(y) & =\mathcal{S}_{1}(0) \theta y^{\theta-1} \exp \left(-\frac{m_{\mathrm{sD}} i}{\bar{\gamma}_{\mathrm{sD}}} y\right) \\
& -\mathcal{S}_{1}(0) \frac{m_{\mathrm{sD}} i}{\bar{\gamma}_{\mathrm{sD}}} y^{\theta} \exp \left(-\frac{m_{\mathrm{sD}} i}{\bar{\gamma}_{\mathrm{sD}}} y\right) .
\end{aligned}
$$

\section{A. General Case}

In $(9), \mathcal{F}_{1}(x)$ can be expressed as

$$
\mathcal{F}_{1}(x)=\int_{I / P_{\max }}^{\infty} f_{Y}(y) F_{\left|h_{\mathrm{sr}}\right|^{2}}\left(\frac{x y}{I}\right) d y .
$$

Assuming also integer values of $m_{\mathrm{sr}}$, substituting (6) and (11) in (12) and employing [14, eq. (3.351/2)], yields the following closed form expression

$$
\begin{aligned}
& \mathcal{F}_{1}(x)=\mathcal{S}_{1}(0) \theta \exp \left(-\frac{m_{\mathrm{sD}} I i}{\bar{\gamma}_{\mathrm{sD}} P_{\max }}\right) \mathcal{S}_{2}(\theta-1,1,1,1, x) \\
& -\mathcal{S}_{1}(1) \exp \left(-\frac{m_{\mathrm{sD}} I i}{\bar{\gamma}_{\mathrm{sD}} P_{\max }}\right) \frac{i m_{\mathrm{sD}}}{\bar{\gamma}_{\mathrm{sD}}} \mathcal{S}_{2}(\theta, 1,1,1, x)
\end{aligned}
$$

where

$$
\begin{gathered}
\mathcal{S}_{2}(\alpha, b, c, d, x)=\sum_{h=0}^{\alpha} \frac{\left(\frac{I}{P_{\max }}\right)^{h} \alpha !}{h !\left(\frac{m_{\mathrm{sD}} i}{\bar{\gamma}_{\mathrm{sD}}}\right)^{\alpha-h+1}}-\sum_{t=0}^{m_{\mathrm{sr}}-1} \frac{1}{t !} \sum_{h=0}^{t+\alpha} \\
\times \frac{\left(\frac{I}{P_{\max }}\right)^{h}}{h !} \frac{(t+\alpha) !}{\left(\frac{m_{\mathrm{sr}} x}{\bar{\gamma}_{\mathrm{sr}} I}\right)^{\alpha-h+1}} \frac{\exp \left(-\frac{m_{\mathrm{ss}} b}{\bar{\gamma}_{\mathrm{sr}} P_{\max }} x\right)}{\left(1+\frac{m_{\mathrm{sD}} i \bar{\gamma}_{\mathrm{sr}} I}{\bar{\gamma}_{\mathrm{sD}} m_{\mathrm{sr}} x}\right)^{d(t+\alpha-h+1)}} c .
\end{gathered}
$$

In addition $\mathcal{F}_{2}(x)$ can be easily evaluated as

$$
\mathcal{F}_{2}(x)=F_{\left|h_{\mathrm{sr}}\right|^{2}}\left(\frac{x}{P_{\max }}\right) F_{Y}\left(\frac{I}{P_{\max }}\right) .
$$

The CDF expression for the output SINR, $\hat{\gamma}_{\mathrm{sr}}$, is given by

$$
F_{\hat{\gamma}_{\mathrm{sr}}}(\gamma)=\int_{0}^{\infty} F_{U}\left[\left(N_{0}+x\right) \gamma\right] f_{Z}(x) d x
$$

where $Z=\sum_{j=1}^{M} P_{T}\left|h_{\mathrm{S}_{j} \mathrm{r}}\right|^{2}$. For i.i.d. fading parameters, and based on the scaling property of the gamma distribution, the PDF of $Z$ can be expressed as follows [17]

$$
f_{Z}(x)=\left(\frac{m_{\mathrm{Sr}}}{P_{T} \bar{\gamma}_{\mathrm{Sr}}}\right)^{m_{\mathrm{Sr}} M} \frac{x^{m_{\mathrm{Sr}} M-1}}{\Gamma\left(m_{\mathrm{Sr}} M\right)} \exp \left(-\frac{m_{\mathrm{Sr}}}{P_{T} \bar{\gamma}_{\mathrm{Sr}}} x\right) .
$$

Substituting (13), (14) and (16) in (15), using the binomial theorem and after some mathematical manipulations yields the closed-form expression for the CDF of the output SINR of the first hop, shown in (17) at the top of the next page ${ }^{1}$. In (17)

$$
\begin{aligned}
& \mathcal{S}_{3}(\alpha, b, c)=\sum_{p_{1}=0}^{t}\left(\begin{array}{c}
t \\
p_{1}
\end{array}\right) \frac{\left(\alpha+\frac{m_{\mathrm{sD}} \bar{\gamma}_{\mathrm{sr}} I i}{\bar{\gamma}_{\mathrm{sD}} m_{\mathrm{sr}} \gamma}\right)^{p_{1}-t-\theta+b-1}}{\left(-\frac{m_{\mathrm{sD}} \bar{\gamma}_{\mathrm{sr}} I i}{\bar{\gamma}_{\mathrm{SD}} m_{\mathrm{sr}} \gamma}\right)^{p_{1}-t}} \\
& \times U\left[m_{\mathrm{Sr}} M ; p_{1}-t-\theta+b ; \frac{\left(\frac{m_{\mathrm{sr}} \gamma}{\bar{\gamma}_{\mathrm{sr}} P_{\max }}+\frac{m_{\mathrm{Sr}}}{P_{T} \bar{\gamma}_{\mathrm{sr}}}\right)}{\left(\alpha+\frac{m_{\mathrm{sD}} \bar{\gamma}_{\mathrm{ss}} I i}{\bar{\gamma}_{\mathrm{sD}} m_{\mathrm{sr}} \gamma}\right)}\right]
\end{aligned}
$$

with $U(\cdot)$ denoting the confluent hypergeometric function [14, eq. $(9.210 / 2)]$.

\section{B. Special Cases}

In order to better understand the impact of the various system parameters on the OP, two special cases will be further investigated. In the first one, the restriction on the available transmission power at the secondary network is neglected, while in the second one an interference limited scenario is investigated.

1) Unrestricted Maximum Transmission Power: We consider the case where $P_{\max } \rightarrow \infty$. For this asymptotic case $P_{\mathrm{s}} \cong I / Y$ and $I$ becomes the main parameter for determining the maximum allowed transmission power [10]. Based on this assumption, $\mathcal{F}_{1}(x)$ can be re-expressed as

$$
\mathcal{F}_{1}(x)=\int_{0}^{\infty} f_{Y}(y) F_{\left|h_{\mathrm{sr}}\right|^{2}}\left(\frac{x y}{I}\right) d y .
$$

Substituting (6) and (11) in (18), employing [14, eq. (3.351/3)], and after some mathematical manipulations, $\mathcal{F}_{1}(x)$, in (13),

${ }^{1}$ Note that $F_{\hat{\gamma}_{\mathrm{rd}}}(\gamma)$ has a similar expression with (17), with sr, $\mathrm{sD}_{i}, \mathrm{~S}_{j} \mathrm{r}$ substituted by $\mathrm{rd}, \mathrm{rD}_{i}, \mathrm{~S}_{j} \mathrm{~d}$, respectively. 


$$
\begin{aligned}
F_{\hat{\gamma}_{\mathrm{sr}}}(\gamma) & =\mathcal{S}_{1}(1) \exp \left(-\frac{I m_{\mathrm{sD}} i}{P_{\mathrm{max}} \bar{\gamma}_{\mathrm{sD}}}\right)\left\{\theta \mathcal{S}_{2}\left[\theta-1, N_{0},\left(\frac{m_{\mathrm{Sr}}}{P_{T} \bar{\gamma}_{\mathrm{Sr}}}\right)^{m_{\mathrm{Sr}} M}, 0, \gamma\right] \mathcal{S}_{3}\left(N_{0}, m_{\mathrm{Sr}} M+h+1,1\right)\right. \\
& \left.-\frac{m_{\mathrm{sD}} i}{\bar{\gamma}_{\mathrm{sD}}} \mathcal{S}_{2}\left[\theta, N_{0},\left(\frac{m_{\mathrm{Sr}}}{P_{T} \bar{\gamma}_{\mathrm{Sr}}}\right)^{m_{\mathrm{Sr}} M}, 0, \gamma\right] \mathcal{S}_{3}\left(N_{0}, m_{\mathrm{Sr}} M+h, 1\right)\right\}+\mathcal{S}_{1}(0)\left(\frac{I}{P_{\mathrm{max}}}\right)^{\theta} \exp \left(-\frac{I m_{\mathrm{SD}} i}{P_{\mathrm{max}} \bar{\gamma}_{\mathrm{sD}}}\right) \\
& \times\left[1-\frac{\exp \left(-\frac{N_{0} \gamma m_{\mathrm{sr}}}{P_{\max } \bar{\gamma}_{\mathrm{sr}}}\right)}{\Gamma\left(m_{\mathrm{Sr}} M\right)}\left(\frac{m_{\mathrm{Sr}}}{P_{T} \bar{\gamma}_{\mathrm{Sr}}}\right)^{m_{S r} M} \sum_{t=0}^{m_{\mathrm{sr}}-1}\left(\frac{m_{\mathrm{sr}} \gamma}{P_{\max } \bar{\gamma}_{\mathrm{sr}}}\right)^{t} \sum_{p_{2}=0}^{t}\left(\begin{array}{c}
t \\
p_{2}
\end{array}\right) \frac{\Gamma\left(m_{\mathrm{Sr}} M+p_{2}\right) N_{0}^{t-p_{2}} / t !}{\left.\left(\frac{m_{\mathrm{sr}} \gamma}{\bar{\gamma}_{\mathrm{sr}} P_{\mathrm{max}}}+\frac{m_{\mathrm{Sr}}}{P_{T} \bar{\gamma}_{\mathrm{Sr}}}\right)^{m_{\mathrm{Sr}} M+p_{2}}\right] .} .\right.
\end{aligned}
$$

simplifies to

$$
\begin{aligned}
& \mathcal{F}_{1}(x)=1-\mathcal{S}_{1}(1) \theta \sum_{t=0}^{m_{\mathrm{sr}}-1} \frac{1}{t !}\left(\frac{m_{\mathrm{sr}} x}{\bar{\gamma}_{\mathrm{sr}} I}\right)^{t} \frac{(t+\theta-1) !}{\left(\frac{m_{\mathrm{sD}} i}{\bar{\gamma}_{\mathrm{sD}}}+\frac{m_{\mathrm{sr}} x}{\bar{\gamma}_{\mathrm{sr}}}\right)^{t+\theta}} \\
& -\mathcal{S}_{1}(1) \frac{m_{\mathrm{sD}} i}{\bar{\gamma}_{\mathrm{sD}}} \sum_{t=0}^{m_{\mathrm{sr}}-1} \frac{1}{t !}\left(\frac{m_{\mathrm{sr}} x}{\bar{\gamma}_{\mathrm{sr}} I}\right)^{t} \frac{(t+\theta) !}{\left(\frac{m_{\mathrm{sD}} i}{\bar{\gamma}_{\mathrm{sD}}}+\frac{m_{\mathrm{sr}} x}{\bar{\gamma}_{\mathrm{sr}} I}\right)^{t+\theta+1}} .
\end{aligned}
$$

Thus substituting (19), (14) and (16) in (15), and using again [18, eq. (2.3.6/6)], yields the simplified closed-form expression of (17) as follows

$$
\begin{aligned}
& F_{\hat{\gamma}_{\mathrm{sr}}}(\gamma)=1-\left(\frac{m_{\mathrm{Sr}}}{P_{T} \bar{\gamma}_{\mathrm{Sr}}}\right)^{m_{\mathrm{Sr}} M}\left[\mathcal{S}_{1}(1) \theta\right. \\
& \times \sum_{t=0}^{m_{\mathrm{sr}}-1} \frac{\Gamma(t+\theta)}{t !\left(\frac{m_{\mathrm{s}} \gamma}{I \overline{\gamma_{\mathrm{sr}}}}\right)^{\theta}} \mathcal{S}_{3}\left(N_{0}, m_{\mathrm{Sr}} M+1,0\right)-\mathcal{S}_{1}(1) i \\
& \left.\times \sum_{t=0}^{m_{\mathrm{sr}}-1} \frac{\Gamma(t+\theta+1)}{t !\left(\frac{m_{\mathrm{sr}} \gamma}{I \bar{\gamma}_{\mathrm{sr}}}\right)^{\theta+1}}\left(\frac{m_{\mathrm{sD}}}{\bar{\gamma}_{\mathrm{sD}}}\right) \mathcal{S}_{3}\left(N_{0}, m_{\mathrm{Sr}} M, 0\right)\right] .
\end{aligned}
$$

2) Interference Limited: In many cases, mobile communication systems tend to be interference limited rather than noise limited, since the thermal and man-made noise effects are insignificant compared to the signal levels of cochannel users [19]. Thus, considering an interference limited environment, i.e., ignoring the AWGN at the user terminal, the received signal-to-interference ratio (SIR) is given by

$$
F_{\hat{\gamma}_{\mathrm{sr}}}(\gamma)=\int_{0}^{\infty} F_{U}(x \gamma) f_{Z}(x) d x
$$

Substituting (13), (14) and (16) in (21) and after some mathematical manipulations yields the CDF of the instantaneous output SIR of the first hop, shown in (22) at the top of the next page. For the asymptotic region of $P_{\max }$, the corresponding expression of the $F_{\hat{\gamma}_{\mathrm{sr}}}(\gamma)$ is given by (20) by replacing $N_{0}$ with 0.

Again, similar to (20) and (22) simplified expressions hold for $F_{\hat{\gamma}_{\mathrm{rd}}}(\gamma)$.

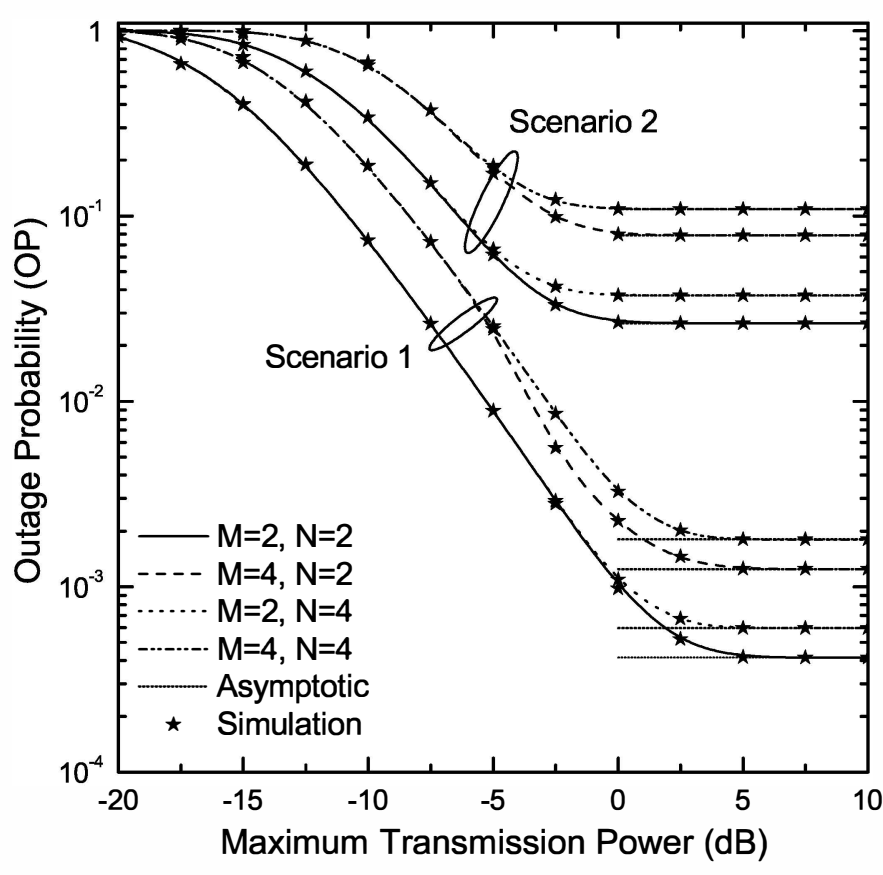

Fig. 2. Outage probability as a function of the maximum transmission power for various numbers of primary users.

\section{NUMERICAL RESULtS}

In this section, using the previously derived expressions for the CDF of the instantaneous output SINR, the OP of the underlay dual-hop DF relay scheme will be studied. For obtaining Fig. 2, we have assumed, a normalized outage threshold $\gamma_{\text {th }} / \bar{\gamma}_{X}=-25 \mathrm{~dB}$, with $X \in\{\mathrm{sr}, \mathrm{rd}\}, I=4 \mathrm{~dB}$, $N_{0}=0 \mathrm{~dB}, P_{T}=1 \mathrm{~dB}, m_{\mathrm{sD}}=3, m_{\mathrm{sr}}=2, m_{\mathrm{Sr}}=2.2$, $M=N \in\{2,4\}$. Under these assumptions and based on (17), the OP is plotted as a function of the maximum allowed transmission power, $P_{\max }$ and for two different communication scenarios. In particular, for Scenario 1 we have considered $\bar{\gamma}_{\mathrm{sD}}=0 \mathrm{~dB}, \bar{\gamma}_{\mathrm{rD}}=1 \mathrm{~dB}, \bar{\gamma}_{\mathrm{Sr}}=1 \mathrm{~dB}, \bar{\gamma}_{\mathrm{Sd}}=2 \mathrm{~dB}$, while for Scenario 2 we have considered $\bar{\gamma}_{\mathrm{sD}}=5 \mathrm{~dB}, \bar{\gamma}_{\mathrm{rD}}=6 \mathrm{~dB}$, $\bar{\gamma}_{\mathrm{Sr}}=6 \mathrm{~dB}, \bar{\gamma}_{\mathrm{Sd}}=7 \mathrm{~dB}$. In the same figure, for comparison purposes, the asymptotic OP, obtained based on (20), is also plotted. It is shown that the performance improves with the increase of $P_{\max }$, reaching in all cases a floor for higher values of $P_{\max }$. The performance also improves for low $M=N$ and for decreased mean values of the channel gains, i.e., Scenario 


$$
\begin{aligned}
F_{\hat{\gamma}_{\mathrm{sr}}}(\gamma) & =\mathcal{S}_{1}(1) \exp \left(-\frac{I m_{\mathrm{sD}} i}{P_{\max } \bar{\gamma}_{\mathrm{sD}}}\right)\left\{\theta\left(\mathcal{S}_{2}\left[\theta-1,0,\left(\frac{m_{\mathrm{Sr}}}{P_{T} \bar{\gamma}_{\mathrm{Sr}}}\right)^{m_{S r} M}, 0, \gamma\right] \mathcal{S}_{3}\left(0, m_{\mathrm{Sr}} M+h+1,1\right)\right)\right. \\
& \left.-\frac{m_{\mathrm{sD}} i}{\bar{\gamma}_{\mathrm{sD}}} \mathcal{S}_{2}\left[\theta, 0,\left(\frac{m_{\mathrm{Sr}}}{P_{T} \bar{\gamma}_{\mathrm{Sr}}}\right)^{m_{\mathrm{Sr}} M}, 0, \gamma\right] \mathcal{S}_{3}\left(0, m_{\mathrm{Sr}} M+h, 1\right)\right\}+\mathcal{S}_{1}(0)\left(\frac{I}{P_{\mathrm{max}}}\right)^{\theta} \\
& \times \exp \left(-\frac{I m_{\mathrm{sD}} i}{P_{\max } \bar{\gamma}_{\mathrm{sD}}}\right)\left[1-\frac{\left(\frac{m_{\mathrm{Sr}}}{P_{T} \bar{\gamma}_{\mathrm{Sr}}}\right)^{m_{S r} M}}{\Gamma\left(m_{\mathrm{Sr}} M\right)} \sum_{t=0}^{m_{\mathrm{sr}}-1}\left(\frac{m_{\mathrm{sr}} \gamma}{P_{\max } \bar{\gamma}_{\mathrm{sr}}}\right)^{t} \frac{\Gamma\left(m_{\mathrm{Sr}} M+t\right) / t !}{\left.\left(\frac{m_{\mathrm{sr}} \gamma}{\bar{\gamma}_{\mathrm{sr}} P_{\max }}+\frac{m_{\mathrm{Sr}}}{P_{T} \bar{\gamma}_{\mathrm{Sr}}}\right)^{m_{\mathrm{Sr}} M+t}\right] .}\right.
\end{aligned}
$$

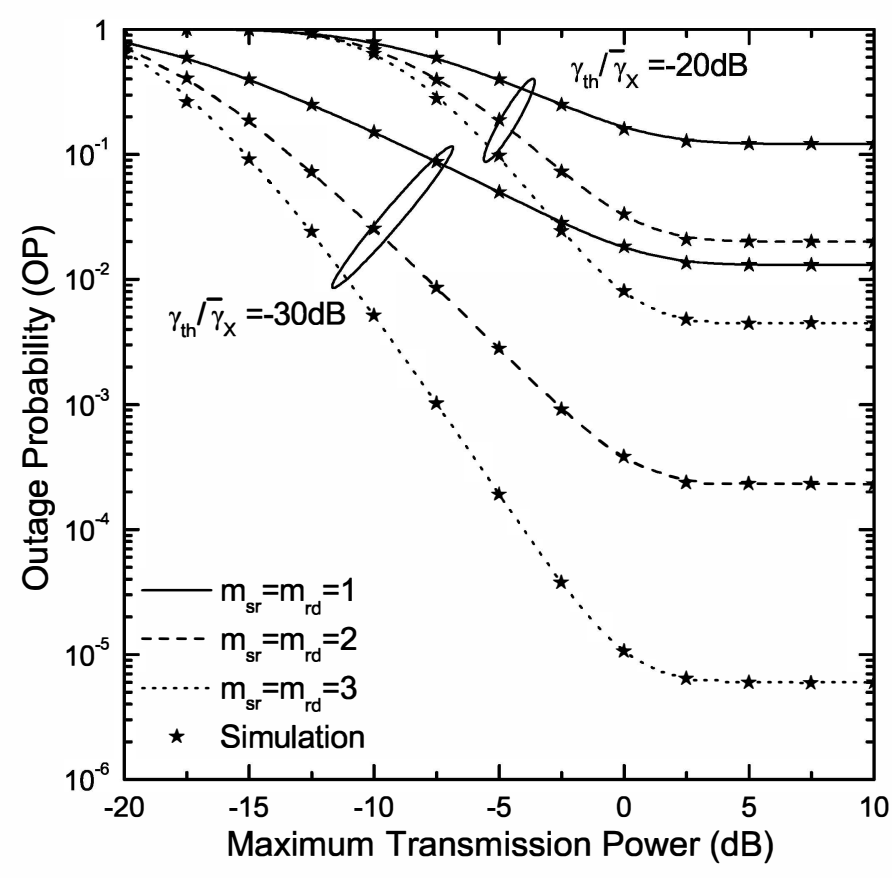

Fig. 3. Outage probability as a function of the maximum transmission power for various values of the normalized outage threshold and different channel conditions.

1. It is interesting to note that the OP is better when $M<N$, as compared to the case $M>N$, which means that the influence of the number of $\mathrm{S}_{j}$ 's is more important than that of $\mathrm{D}_{i}$ 's for these communication scenarios. Another interesting observation is that the asymptotic expressions for the OP are quite tight, especially for higher values of $P_{\max }$.

In Fig. 3, in order to highlight the impact of fading channel conditions to the system's performance, the OP is plotted as a function of $P_{\max }$, for different values of $m_{\mathrm{X}}$ and $\gamma_{\mathrm{th}} / \bar{\gamma}_{X}$. For obtaining Fig. 3, we have assumed Scenario 1 and the same values for $I, N_{0}, P_{T}$ as in Fig. 2, and $m_{\mathrm{Sr}}=m_{\mathrm{Sd}}=m_{\mathrm{sD}}=$ $m_{\mathrm{rD}} \cong 2$. It is depicted that the performance improves for higher values of the normalized threshold and the maximum transmission power. In addition, in all cases, the performance improves for higher values of the s-r-d shaping parameters, i.e., better channel conditions. Further research attempts have shown that for asymptotically increased values of $m_{X}$ the performance gain is very limited.

Finally, in Fig. 4, we focus on the interference limited case, where we have assumed $P_{T}=5 \mathrm{~dB}, m_{\mathrm{sD}}=2, m_{\mathrm{sr}}=$ $3, m_{\mathrm{Sr}} \cong 2, M=N=3, P_{\max }=10 \mathrm{~dB}$. Under these assumptions, the OP is plotted as a function of $\gamma_{\mathrm{th}} / \bar{\gamma}_{X}$ for different scenarios and values of the maximum tolerable interference level $I$. In particular, we have assumed: Scenario 1: $\bar{\gamma}_{\mathrm{sD}}=\bar{\gamma}_{\mathrm{rD}}=\bar{\gamma}_{\mathrm{Sr}}=\bar{\gamma}_{\mathrm{Sd}}=0 \mathrm{~dB}$, Scenario 2: $\bar{\gamma}_{\mathrm{sD}}=\bar{\gamma}_{\mathrm{rD}}=$ $0 \mathrm{~dB}, \bar{\gamma}_{\mathrm{Sr}}=\bar{\gamma}_{\mathrm{Sd}}=5 \mathrm{~dB}$, Scenario 3: $\bar{\gamma}_{\mathrm{sD}}=\bar{\gamma}_{\mathrm{rD}}=5 \mathrm{~dB}$, $\bar{\gamma}_{\mathrm{Sr}}=\bar{\gamma}_{\mathrm{Sd}}=0 \mathrm{~dB}$ and Scenario 4: $\bar{\gamma}_{\mathrm{sD}}=\bar{\gamma}_{\mathrm{rD}}=\bar{\gamma}_{\mathrm{Sr}}=$ $\bar{\gamma}_{\mathrm{Sd}}=5 \mathrm{~dB}$. It is depicted that the performance improves as $I$ increases, i.e., the primary network's interference tolerance loosens. Additionally, the performance improves for lower channel gains mean values. However, increasing $\bar{\gamma}_{\mathrm{Sr}}=\bar{\gamma}_{\mathrm{Sd}}$ improves more the system performance, as compared to the increase of $\bar{\gamma}_{\mathrm{sD}}=\bar{\gamma}_{\mathrm{rD}}$. For comparison purposes, computer simulation performance results are also included in all figures, verifying the validity of the proposed theoretical approach.

\section{CONCLUSIONS}

In this paper the performance of an underlay CRN operating over Nakagami- $m$ fading in the presence of multiple primary users interference, is investigated. Exact as well as approximated expressions of the CDF of the output SINR have been derived and used to evaluate the OP. Various numerical evaluated results with parameters of interest the severity of the fading, the number of primary users, the interference limit and the maximum allowed transmission power have been presented. In all cases, it was shown that the performance worsens as the interference constraint becomes tighter and/or the secondary channel conditions become worse and/or the number of primary users increases.

\section{ACKNOWLEDGEMENT}

This research has been co-financed by the European Union (European Social Fund - ESF) and Greek national funds through the Operational Program "Education and Lifelong Learning" of the National Strategic Reference Framework (NSRF) - Research Funding Program: Thales. Investing in knowledge society through the European Social Fund. 


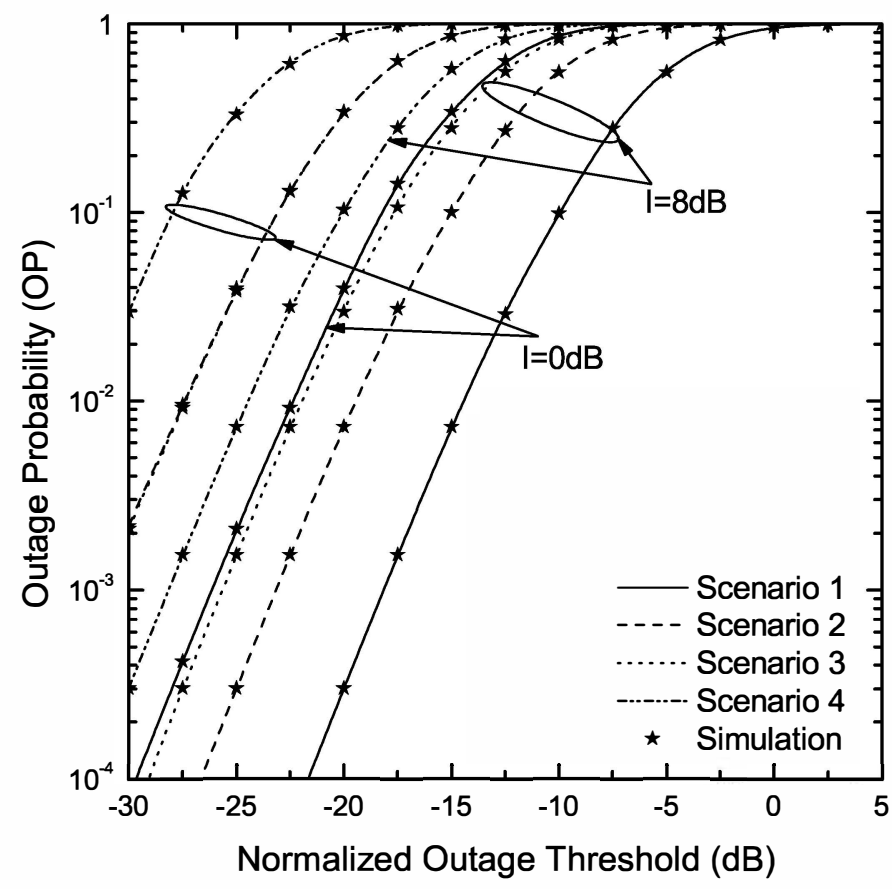

Fig. 4. Outage probability as a function of the normalized outage threshold for various values of the channel gains mean values and the maximum tolerable interference level.

\section{REFERENCES}

[1] A. Goldsmith, S. A. Jafar, I. Maric, and S. Srinivasa, "Breaking spectrum gridlock with cognitive radios: An information theoretic perspective," Proceedings of the IEEE, vol. 97, no. 5, pp. 894-914, May 2009.

[2] T. Q. Duong, D. Benevides da Costa, M. Elkashlan, and V. N. Q. Bao, "Cognitive amplify-and-forward relay networks over Nakagami-fading," IEEE Trans. Veh. Technol., vol. 61, no. 5, pp. 2368-2374, Jun. 2012.

[3] J. Lee, H. Wang, J. G. Andrews, and D. Hong, "Outage probability of cognitive relay networks with interference constraints," IEEE Trans. Commun., vol. 10, no. 2, pp. 390-395, Jul. 2011.

[4] W. Xu, J. Zhang, P. Zhang, and C. Tellambura, "Outage probability of decode-and-forward cognitive relay in presence of primary user's interference," IEEE Commun. Lett., vol. 16, no. 8, pp. 1252-1255, Aug. 2012.

[5] M. Xia and S. Aissa, "Cooperative AF relaying in spectrum-sharing systems: outage probability analysis under co-channel interferences and relay selection," IEEE Trans. Commun., vol. 60, no. 11, pp. 3252-3262, Nov. 2012.

[6] J. Si, Z. Li, H. Huang, J. Chen, and R. Gao, "Capacity analysis of cognitive relay networks with the PU's interference," IEEE Commun Lett., vol. 16, no. 12, pp. 2020-2023, Dec. 2012.

[7] T. Xu, J. Ge, and H. Ding, "An efficient distributed link selection scheme for AF-based cognitive selection relaying networks," IEEE Commun. Lett., vol. 18, no. 2, pp. 253-256, Feb. 2014.

[8] T. Duong, P. L. Yeoh, V. N. Q. Bao, M. Elkashlan, and N. Yang, "Cognitive relay networks with multiple primary transceivers under spectrum-sharing," IEEE Signal Process. Lett., vol. 19, no. 11, pp. 741744, Nov. 2012.

[9] L. Fan, X. Lei, T. Duong, R. Hu, and M. Elkashlan, "Multiuser cognitive relay networks: Joint impact of direct and relay communications," accepted for publication, 2014.

[10] C. Zhong, T. Ratnarajah, and K.-K. Wong, "Outage analysis of decodeand-forward cognitive dual-hop systems with the interference constraint in Nakagami-fading channels," IEEE Trans. Veh. Technol., vol. 60, no. 6, pp. 2875-2879, Jul. 2011.

[11] F. Xu, F. Lau, Q. Zhou, and D.-W. Yue, "Outage performance of cooperative communication systems using opportunistic relaying and selection combining receiver," IEEE Signal Process. Lett., vol. 16, no. 4, pp. 237-240, Apr. 2009.

[12] J. Wang, Z. Zhang, Q. Wu, and Y. Huang, "Outage analysis of cognitive relay networks with interference constraints in Nakagami-m channels," IEEE Wireless Commun. Lett., vol. 2, no. 4, pp. 387-390, Aug. 2013.

[13] A. Ghasemi and E. Sousa, "Fundamental limits of spectrum-sharing in fading environments," IEEE Trans. Wireless Commun., vol. 6, no. 2, pp. 649-658, Feb. 2007.

[14] I. S. Gradshteyn and I. M. Ryzhik, Table of Integrals, Series, and Products, 6th ed. New York: Academic Press, 2000.

[15] T. Q. Duong, V. N. Q. Bao, and H. Zepernick, "On the performance of selection decode-and-forward relay networks over Nakagami-m fading channels," IEEE Commun. Lett., vol. 13, no. 3, pp. 172-174, Mar. 2009.

[16] M. Abramowitz and I. A. Stegun, Handbook of Mathematical Functions, with Formulas, Graphs, and Mathematical Tables, 9th ed. New York: Dover, 1972.

[17] S. Ross, Probability Models, 8th ed. Amsterdam: Academic, 2003.

[18] A. Prudnikov, Y. Brychkov, and O. Marichev, Integrals and Series, Volume 2. Gordon and Breach Science Publishers, 1986.

[19] J. B. Andersen, T. S. Rappaport, and S. Yoshida, "Propagation measurements and models for wireless communications channels," IEEE Commun. Mag., vol. 33, no. 1, pp. 42-49, Jan. 1995. 